\title{
Primary hyperparathyroidism - a diagnostic approach
}

\author{
S Pathmanathan ${ }^{1}$, Noel P Somasundaram ${ }^{2}$ \\ Sri Lanka Journal of Diabetes, Endocrinology and Metabolism 2012; 2: 101-105
}

\begin{abstract}
Primary hyperparathyroidism (PHPT) is characterized by the autonomous production of parathyroid hormone (PTH), in which there is hypercalcemia or normal-high serum calcium levels, in the presence of elevated or inappropriately normal serum PTH concentrations. Diagnosis of PHPT is biochemical. Advances in imaging technology, intraoperative parathyroid hormone measurement, and surgical technique now allow parathyroidectomy to be performed using a focused approach without the absolute need of a fourgland exploration. This brief review summarizes the various diagnostic modalities available for successful preoperative localization and management of the modern day PHPT patient.
\end{abstract}

\section{Introduction}

Primary hyperparathyroidism (PHPT) occurs as a result of increased and uncontrolled secretion of parathyroid hormone because of hyperfunction of one or more parathyroid glands. The cause of hyperfunction of parathyroid glands is, in the majority of cases, an adenoma/ multiple adenomata, followed by hyperplasia in 1 to $15 \%$ of patients, and carcinoma only in 1 to $2 \%$ of cases. Adenomas may be found in ectopic locations in about $16 \%$ of cases - commonly the thymus, tracheaoesophageal groove, mediastinum and the thyroid. The frequency of primary hyperparathyroidism is 1-4/1000 individuals in the general population $(1,2)$. Women are twice as likely to be affected as men, and the commonest age of presentation in between 50 and 60 years of age (3).

With increased detection by means of routine calcium screening, the clinical profile of primary hyperparathyroidism in Western countries has shifted from a symptomatic disease, characterized by hypercalcemic symptoms, nephrolithiasis, overt bone disease, and neuromuscular symptoms to one with subtle or no specific symptoms ("asymptomatic" primary hyperparathyroidism) $(2,3,4)$. In the developing world, the symptomatic variant still dominates (6). In our part of the world where serum calcium is not measured as part of the routine screening, PHPT must always be evaluated in patients with clinical histories of nephrolithiasis, nephrocalcinosis, osseous pain, subperiosteal resorption, and pathologic fractures, as well as in those with age inappropriate osteoporosis-osteopenia on dual-energy Xray absorptiometry (DXA) (6,7). Evaluation may be also useful in patients with resistant dyspepsia or chronic vague gastrointestinal symptoms.

\section{Diagnosis of primary hyperparathyroidism}

\section{Laboratory diagnosis}

The diagnosis of hyperparathyroidism is usually first suspected because of the finding of an elevated serum calcium concentration. If hypercalcemia is confirmed on a repeat sample, the serum parathyroid hormone (PTH) concentration should then be measured. The diagnosis of primary hyperparathyroidism is usually made by finding a frankly elevated PTH concentration or one that is within the normal range but inappropriately elevated given the patient's hypercalcemia. A 24-hour urine calcium measurement is necessary to rule out familial hypocalciuric hypercalcemia (FHH). Other laboratory findings include mild hyperchloremic acidosis, hypophosphatemia, increased alkaline phosphatase and mild-to-moderate increase in urinary calcium and inorganic phosphorus excretion rate $(1,2,3,7)$.

\section{Parathyroid hormone (PTH)}

Parathyroid hormone (PTH) is a single-chain polypeptide containing 84 amino acids. It exerts its effects through the interaction of its first 34 amino acids with the type $1 \mathrm{PTH} / \mathrm{PTHrP}$ receptor (PTHR1). PTH has a plasma half-life of two to four minutes. PTH undergoes proteolysis to yield $\mathrm{N}$-terminal fragments and longer lived C-terminal and mid region fragments. The $\mathrm{N}$-terminal fragment contains the region that confers bioactivity. Generally less than 5 to $25 \%$ of total immunoreactive PTH is intact hormone. The remaining 75 to 95\% is inactive midregion/ carboxyl fragments. The first generation assays included the whole PTH molecule. Second generation assays (intact PTH) measure the active PTH (35-84), which is actually the fragment of PTH present in highest amounts in blood 
and also PTH (7-84) as well. In most people, this fragment is present in much lower amounts than PTH (1-84), so this is not a concern. In kidney failure, a common setting for measuring PTH levels, PTH (7-84) levels increase compared to PTH (1-84), and sometimes over half of what is measured as PTH represents this $\mathrm{N}$-terminal truncated fragment. To overcome this problem the newer 3rd generation (Bio-intact PTH (BI-PTH)) assays has come into practice (8).

BI-PTH by chemiluminescence eliminates interference from inactive PTH fragments, specifically the 7-84 PTH fragments and offers improved sensitivity and specificity to diagnose secondary hyperparathyroid disease in individuals with early and end-stage renal disease. There is no overall difference between secondand third-generation assays for the diagnostic evaluation of PHPT; however, both of these newer generation assays represent an improvement over the first-generation PTH assay (8).

\section{Problems in the diagnosis of PHPT}

\section{Familial hypocalciuric hypercalcemia}

Familial hypocalciuric hypercalcemia $(\mathrm{FHH})$ is a rare disorder that can also present with hypercalcemia and mildly elevated or inappropriately normal PTH levels, and as a result it must be carefully distinguished from PHPT. $\mathrm{FHH}$ is autosomal dominant in inheritance and in the majority of cases is due to a heterozygous mutation in the calcium-sensing receptor (CaSR) gene, the main regulator of parathyroid cell response to calcium. The diagnosis of heterozygous FHH is confirmed by the measurement of the calcium/creatinine clearance ratio $(\mathrm{Ca} / \mathrm{Cr})$. In FHH the $\mathrm{Ca} / \mathrm{Cr}$ clearance ratio is usually less than 0.01 . In pHPT the $\mathrm{Ca} / \mathrm{Cr}$ clearance ratio is typically greater than 0.02 (9).

\section{Normocalcemic hyperparathyroidism}

Hypercalcemia is not always present in all patients with PHPT. For some patients the serum calcium may be at the upper end of the normal range in association with inappropriate elevation of the PTH. This condition is called normocalcemic or subclinical HPT. Two observational studies of normocalcemic PHPT have shown that $19 \%$ will go on to develop classic primary hyperparathyroidism. However, 40\% developed evidence of disease progression with development of kidney stones, fractures, marked hypercalciuria or $>10 \%$ decline in BMD. In the differential diagnosis of patients with normocalcemia and elevation of PTH, potential causes of secondary hyperparathyroidism should also be ruled out. Thus, the possibilities of renal insufficiency, vitamin D deficiency or hypercalciuria must be evaluated. If vitamin D deficiency is suspected a trial of calcium and vitamin D supplements can markedly reduce PTH levels and may preclude unnecessary surgery (10).

\section{Preoperative localization}

Localization techniques were originally used primarily to search for ectopic parathyroid tissue in patients with recurrent or persistent hyperparathyroidism after unsuccessful neck exploration. Currently, with the increased popularity of minimally invasive surgery, parathyroid localization is required to determine whether or not patients are candidates for this approach. It is important to emphasize, however, that preoperative localization studies are only performed to help plan the operative approach. They should not be used to diagnose or confirm the diagnosis of primary hyperparathyroidism. Localization studies should therefore be reserved for patients in whom the biochemical diagnosis of primary hyperparathyroidism is secure. Localization of abnormal parathyroid glands preoperatively can reduce operative time, postoperative morbidity and the requirement for repeat surgery. Imaging techniques for localizing abnormal parathyroid glands and guiding in surgical management include bone densitometry, high resolution ultrasonography (USG), CT, MRI and the radionuclide imaging. Plain skeletal radiography is not routinely recommended in the diagnosis except in resource poor settings where they can favour the diagnosis $(11,12)$.

\section{Imaging techniques}

\section{Bone densitometry and plain skeletal radiography}

PTH has a catabolic effect on cortical bone, and sites enriched in cortical bone are preferentially reduced. Most patients have reduced bone mineral density at the distal third of the forearm with relative preservation at the lumbar spine and intermediate values at the hip. Plain radiographic findings include resorption and sclerosis of the middle phalanges of the index and middle fingers (primarily on the radial aspect), phalangeal tufts (acro-osteolysis), the lamina dura around the teeth, the medial aspect of the tibia, the humerus, the femur, and the distal clavicle. In cases of severe primary hyperparathyroidism, skeletal radiographs show pathognomonic changes such as salt-and-pepper degranulation in the skull and brown tumours of the cortical bones. Common sites include the mandible, clavicle, ribs, pelvis, and femur. Plain skeletal radiography is not routinely recommended except in very severe cases $(11,12)$.

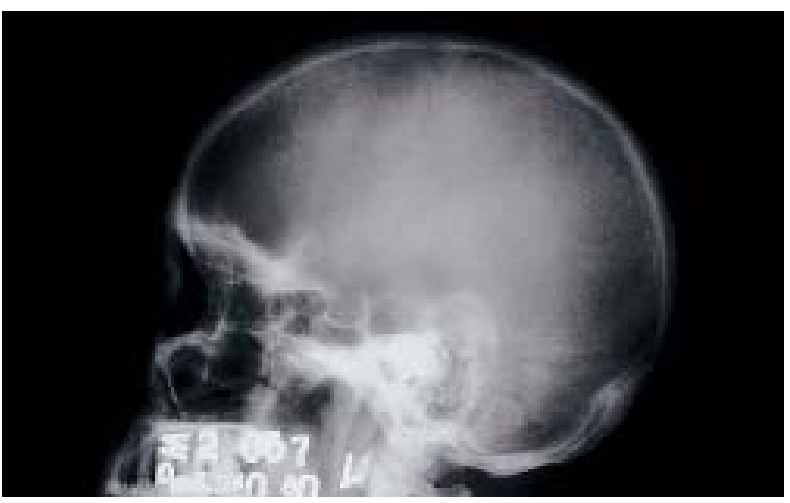

Figure 1. Pepper pot skull. 


\section{High resolution USG}

High resolution USG is one of the most common imaging methods used for neck evaluation and it is practically the first option in the primary hyperparathyroidism assessment. On USG, parathyroid adenoma is seen typically as round or oval homogenous, hypoechoic nodule localized behind the thyroid gland and at the lower aspect of paratracheal or paraeosophageal region. It is clearly separated from thyroid gland due to its capsule. Morphological differences such as hyperechoic component, cystic changes and calcification may be seen particularly in large adenomas. More than $90 \%$ of parathyroid adenomas include intraparenchymal hypervascular pattern in the color flow imaging (8). Ultrasonography offers the advantage of depicting potential concomitant thyroid disease, which is present in approximately $40 \%$ of patients with parathyroid disease. Ultrasonography is approximately $75 \%$ sensitive in identifying adenomas, but this technique has low sensitivity in identifying ectopic lesions (13).

\section{CT}

Standard CT scanning has inadequate sensitivity. Newer techniques of CT scanning with dynamic contrast images (4D-CT) have shown promise, with accuracy rates as high as $88 \%$. One of the advantages of CT over USG is its ability to determine particularly ectopic parathyroid adenomas in the mediastinum $(13,14)$.

\section{MRI}

Sensitivity of MR in the determination of parathyroid adenoma varies between 65-80\%. On T1-weighted images, adenomas appear as low-signal-intensity masses, whereas intermediate or high signal intensity is seen on T2weighted images. MRI can be useful, particularly in cases of recurrent or persistent disease and in ectopic locations such as the mediastinum $(13,15)$.

\section{Radionuclide imaging of the parathyroid glands}

Parathyroid scintigraphy remains an important tool for guiding clinical and surgical decisions. Sestamibi with 99mTc is the most commonly used radiotracer for imaging the parathyroid glands and has been extensively studied in the setting of primary hyperparathyroidism. Sestamibi is taken up by both the thyroid and parathyroid glands. It clears from the thyroid with a half-life of about 30 minutes but is usually retained by abnormal parathyroid glands. In dual-phase planar imaging, the thyroid and parathyroid glands are imaged at 5 minutes after tracer injection; images are repeated at 2 hours. Initial images will show both thyroid and parathyroid tissue whereas on delayed images, an abnormal parathyroid is seen as a persistent focus of activity. The scan's sensitivity for detecting solitary adenomas has varied widely in the literature but generally is reported as $60-90 \%$. The main weakness of this test is in diagnosing multiglandular disease. In this case, sensitivity drops to approximately $50 \%$. When combined with singlephoton emission computed tomography (SPECT) scanning, it can be used effectively to localize parathyroid adenomas. The scan can include the mediastinum and, thus, is extremely useful in cases of an ectopic adenoma or previously failed surgical exploration. Various studies have shown that 99mTc-sestamibi and 99mTc-tetrofosmin have equal sensitivity for the localization of abnormal parathyroid glands $(15,16)$. Dual-tracer subtraction scintigraphy combines dual-phase 99mTc-tetrofosmin with administration of a second radiopharmaceutical that accumulates specifically in the thyroid gland and not in the parathyroid tissue; images are then subtracted to allow detection of focal uptakes specific for abnormal parathyroid tissue. This study is not found to be superior to 99mTc- sestamibi or 99mTc-tetrofosmin scanning (17). In Sri Lanka 99mTc-tetrofosmin scanning is available in the government sector where as $99 \mathrm{mTc}$-Sestamibi scans are available in the private sector which would cost about 24,000 LKR (Figure 2).

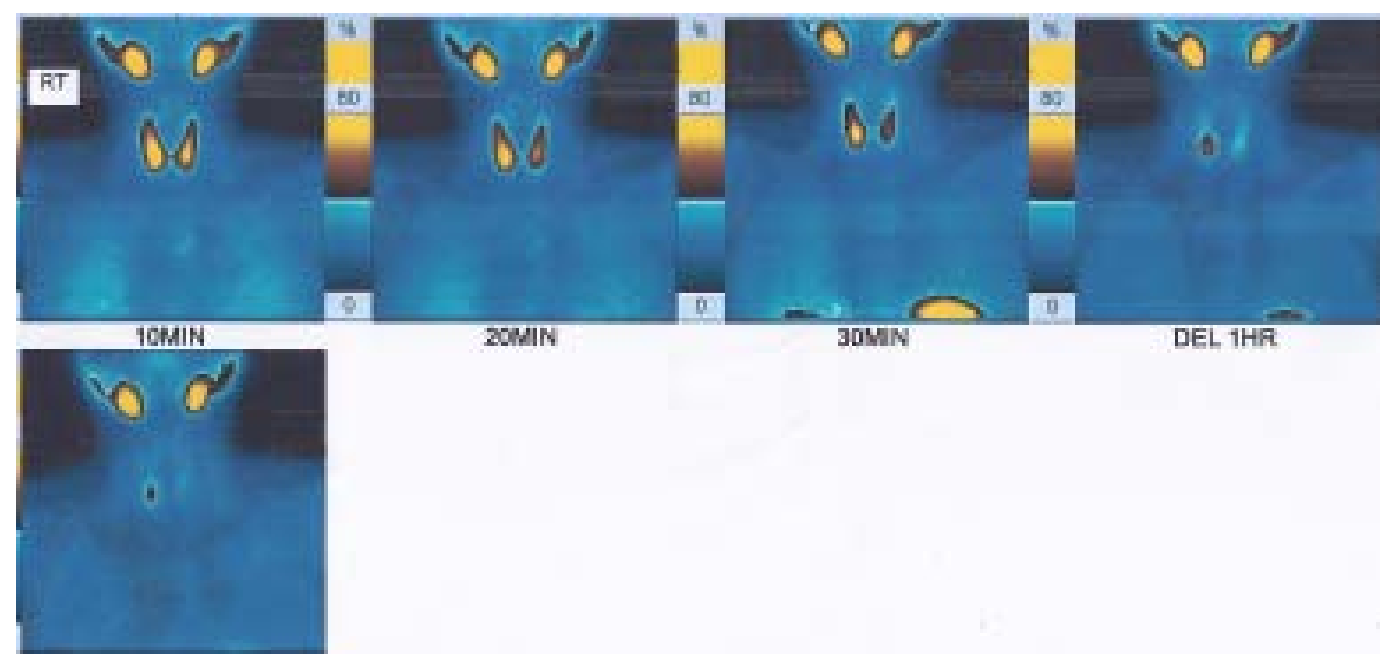

Figure 2. Sestamibi scan showing right parathyroid adenoma. 


\section{Selective venous sampling of the parathyroids}

Selective venous sampling and PTH measurements are performed to determine the general location of a parathyroid adenoma. A parathyroid arteriogram should be performed first because this serves as a guide or road map to the more variable parathyroid venous pathways. An end-hole catheter without side holes should be used to prevent the mixing of blood from adjacent veins. Sampling of small veins is the goal. After each sample is obtained, a small amount of contrast material is injected, and a spot image is obtained to document the location of the catheter tip and sampling site. Lastly, a peripheral vein sample is obtained. A 2-fold gradient between the PTH concentration in the sampled vein and that of the peripheral vein is considered to be diagnostic. With modifications, this technique has also been used during surgery to confirm success in removing the source of increased PTH production. The sensitivity of parathyroid venous sampling is $70-80 \%(18,19)$.

\section{Parathyroid fine-needle aspiration (FNA) with parathyroid hormone washout}

Ultrasound guided parathyroid fine needle aspiration was first described by Doppman et al in 1983. It became more frequently used during the late 1990s and today it is a novel, reproducible, and highly successful method of preoperative localization suitable for focused parathyroidectomy. This technique is almost restricted to reoperative patients. In a reoperative setting when scarring, distortion of anatomic landmarks, and a higher number of ectopic parathyroid glands in this subgroup make another intervention more difficult, correct localization is pivotal. It allows identification of parathyroid adenoma via a minimally invasive approach, especially in cases where a sestamibi scan is inconclusive. FNA is performed under USG or CT guidance followed by a washout procedure using isotonic saline. Then the blood tinged fluid is submitted for PTH assay. PTH $>1,000 \mathrm{pg} / \mathrm{ml}$ in the needlewashing fluid is considered positive. With newer techniques different institutions are using different cutoff values. So these values need to be revalidated in future. This procedure has a sensitivity and specificity of 91\%100\%. The main limitation of parathyroid FNA with PTH washout are is its dependence on identification of a suspicious lesion by USG or CT and the number of false negative results (20).

\section{Intraoperative PTH(IOPTH) monitoring}

PTH monitoring takes advantage of the short halflife (three to five minutes) of PTH and utilizes a rapid immunochemiluminescence assay technique that allows measurements while the patient is still in the operating room. A drop in rapid PTH levels of greater than $50 \%$ at 10 minutes after excision of hyperfunctioning tissue is predictive of postoperative normocalcemia in patients with hyperparathyroidism. Patients with no drop in intraoperative PTH levels have generally remained hypercalcemic immediately after surgery. Numerous studies have shown that rapid intraoperative PTH testing, in the setting of primary hyperparathyroidism, is accurate in predicting surgical success. Cure rates of $>95 \%$ have been reported in several studies. However, several socalled false-negative results have been reported where a delayed (up to 30 minutes) drop in PTH levels has occurred and there have been complete cure. In addition, rapid PTH levels may initially rise within the first few minutes after excision of a hyperfunctioning gland, possibly because of manipulation of parathyroid tissue with augmented systemic release of PTH into the bloodstream before excision (21).

Rapid IOPTH monitoring is particularly useful in reoperative parathyroidectomy. Combined use of the rapid PTH assay with preoperative Sestamibi localization may prevent unwanted dissection of previously operated patients who have recurrent or uncured disease. The ability of the rapid PTH assay to detect the presence of multiglandular parathyroid hyperplasia is unclear. Some studies have suggested that IOPTH levels will typically fall in a sequential manner as each of the hyperfunctioning glands is removed. IOPTH monitoring is extremely costly even in the best centers and it is not available in Sri Lanka.

\section{Conclusion}

Diagnosis of PHPT is straight forward. Preoperative localization is not necessary in the traditional four gland exploration. Preoperative localization studies are only performed to help plan the operative approach and they should not be used to diagnose or confirm the diagnosis of primary hyperparathyroidism. Ultrasonography and Sestamibi scanning are used commonly. The optimal preoperative localization technique is best decided on local availability in consultation with an experienced surgeon or who has done a large number of parathyroid surgeries.

\section{References}

1. Fraser WD. Hyperparathyroidism. Lancet 2009; 374(9684): 145-58.

2. Marcocci C, Cetani F. Primary hyperparathyroidism. New England Journal of Medicine 2011; 365: 2389-97.

3. Pallan S, Rahman MO, Khan AA. Diagnosis and management of primary hyperpara-thyroidism. British Medical Journal 2012; 344: 55-60.

4. Wermers RA, Khosla S, Atkinson EJ, Hodgson SF, O’Fallon WM, Melton LJ. The rise and fall of primary hyperparathyroidism: a population-based study in Rochester, Minnesota, 1965-1992. Annals of Internal Medicine 1997; 126: 433-40. 
5. Pallan S. Primary hyperparathyroidism - update on presentation, diagnosis, and management in primary care. Canadian Family Physician 2011; 57: 184-9.

6. Bhansali A, Masoodi SR, Reddy KSS. Primary hyperparathyroidism in north India: a description of 52 cases. Annals of Saudi Medicine 2005; 25(1): 29-35.

7. Suliburka JW, Perrierb ND. Primary hyperparathyroidism. The Oncologist 2007; 12: 644-53.

8. Goodman WG. New assays for parathyroid hormone (PTH) and the relevance of PTH fragments in renal failure. Kidney International 2003; 87: S120-S124.

9. Heath H. Familial benign (hypocalciuric) hypercalcemia. A troublesome mimic of mild primary hyperparathyroidism. Endocrinology and Metabolism Clinics of North America 1989; 18: 723-40.

10. Cetani F. Normocalcemic primary hyperparathyroidism Endocrine Abstracts 2011; 26: S4.2.

11. Ahuja AT, Wong KT, Ching AS, et al. Imaging for primary hyperparathyroidism-what beginners should know. Clinical Radiology 2004; 59: 967-76.

12. Gotway MB, Leung JWT, Gooding GA, et al. Hyperfunctioning parathyroid tissue: spectrum of appearances on noninvasive imaging. American Journal of Roentgenology 2002; 179: 495-502.

13. Tziakouri C, Eracleous E, Skannavis S, et al. Value of ultrasonography, CT and MR imaging in the diagnosis of primary hyperparathyroidism. Acta Radiologica 1996; 37: 720-6.
14. Mazzeo S, Cappelli C, Caramella D et al. Multidetector CT in diagnostic work-up of patients with primary hyperparathyroidism. La Radiologia Medica 2007; 112(5): 763-775.

15. Ishibashi M, Nishida H, Hiromatsu Y, Kojima K, Tabuchi E, Hayabuchi N. Comparison of Technetium-99m-MIBI, Technetium-99m-Tetrofosmin, ultrasound and MRI for localization of abnormal parathyroid glands. Journal of Nuclear Medicine 1998; 39(2): 320-4.

16. Joel RM, Oates E, Smith. Radionuclide imaging of the parathyroid glands: patterns, pearls, and pitfalls. Radio Graphics 2004; 24: 1101-15.

17. Perez-Monte JE, Brown ML, Shah AN, Ranger NT, Watson CG, Carty SE. Parathyroid adenomas: accurate detection and localization with Tc-99m sestamibi SPECT. Radiology 1996; 201: 85-91.

18. Miller DL. Endocrine angiography and venous sampling. Radiologic Clinics of North America 1993; 31(5): 1051-67.

19. Chaffanjon PC, Voirin D, Vasdev A, et al. Selective venous sampling in recurrent and persistent hyperparathyroidism: indication, technique, and results. World Journal of Surgery 2004; 28(10): 958-61.

20. Bancos I, Grant CS, Nadeem S, et al. Risks and benefit of parathyroid fine-needle aspiration with parathyroid hormone washout. Endocrine Practice 2012; 18(4): 44150.

21. Mandell DL, Eric M, et al. The influence of intraoperative parathyroid hormone monitoring on the surgical management of hyperparathyroidism. Archives of Otolaryngology Head and Neck Surgery 2001; 127(7): 821-7. 